\title{
Regulation of pluripotency and differentiation by deubiquitinating enzymes
}

\author{
B Suresh ${ }^{1,5}, \mathrm{~J} \mathrm{Lee}^{2,5}, \mathrm{H} \mathrm{Kim}^{\star, 1}$ and S Ramakrishna ${ }^{\star, 3,4}$
}

Post-translational modifications (PTMs) of stemness-related proteins are essential for stem cell maintenance and differentiation. In stem cell self-renewal and differentiation, PTM of stemness-related proteins is tightly regulated because the modified proteins execute various stem cell fate choices. Ubiquitination and deubiquitination, which regulate protein turnover of several stemnessrelated proteins, must be carefully coordinated to ensure optimal embryonic stem cell maintenance and differentiation. Deubiquitinating enzymes (DUBs), which specifically disassemble ubiquitin chains, are a central component in the ubiquitinproteasome pathway. These enzymes often control the balance between ubiquitination and deubiquitination. To maintain stemness and achieve efficient differentiation, the ubiquitination and deubiquitination molecular switches must operate in a balanced manner. Here we summarize the current information on DUBs, with a focus on their regulation of stem cell fate determination and deubiquitinase inhibition as a therapeutic strategy. Furthermore, we discuss the possibility of using DUBs with defined stem cell transcription factors to enhance cellular reprogramming efficiency and cell fate conversion. Our review provides new insight into DUB activity by emphasizing their cellular role in regulating stem cell fate. This role paves the way for future research focused on specific DUBs or deubiquitinated substrates as key regulators of pluripotency and stem cell differentiation. Cell Death and Differentiation (2016) 23, 1257-1264; doi:10.1038/cdd.2016.53; published online 10 June 2016

Facts

- Ubiquitination and deubiquitination of stemness-related proteins are well coordinated to ensure optimal embryonic stem cell maintenance and differentiation.

- Extensive research has been achieved on ubiquitination system in the maintenance of stem cell and differentiation. Deubiquitinating enzymes (DUBs)-mediated reversal of ubiquitination also has an equally critical role.

- Recent studies with USP7, USP9X, USP22, USP44, and Psmd14 have shown that DUBs are involved in maintaining stem cell pluripotency.

- First attempt to review the relationship between DUBs and stem cells, and suggesting DUBs as potential candidates for regulating stem cell fate determination and cellular reprogramming.

\section{Open Questions}

- What is the evidence to support the involvement of DUBs in stem cells?
- What is the role of DUBs in regulating stem cell fate determination?

- How can the DUBs be targeted to regulate stem cell pluripotency, differentiation, and cellular reprograming?

Embryonic stem cells (ESCs) that are derived from the inner cell mass (ICM) of the blastocyst can undergo unlimited selfrenewal. Moreover, ESCs can be triggered to differentiate into all three embryonic germ layers: (a) ectoderm - skin and nerve; (b) mesoderm - bone, blood, and muscle; and (c) endoderm - gut and lung tissues. Human ESCs were first isolated by Thomson et al. ${ }^{1}$ from the ICM of preimplantation blastocysts.

ESC self-renewal and differentiation are known to be regulated by a network of transcription factors including Oct3/4, Sox2, c-Myc, Klf4, and Nanog. ${ }^{2,3}$ However, in addition to transcription-mediated regulation of ESC fate, recent studies have indicated that transcription-independent mechanisms also exist for controlling ESC fate. However, little information has been obtained regarding the role of posttranscriptional modifications (PTMs) in ESC maintenance and differentiation.

\footnotetext{
${ }^{1}$ Department of Pharmacology and Brain Korea 21 PLUS Project for Medical Science, Yonsei University College of Medicine, Seoul, South Korea; ${ }^{2}$ Department of Physiology and Brain Korea 21 PLUS Project for Medical Science, Yonsei University College of Medicine, Seoul, South Korea; ${ }^{3}$ Graduate School of Biomedical Science and Engineering, Department of Biomedical Science, Hanyang University, Seoul, South Korea and ${ }^{4}$ College of Medicine, Department of Biomedical Science, Hanyang University, Seoul, South Korea

${ }^{*}$ Corresponding author: H Kim. Department of Pharmacology, Yonsei University College of Medicine, Seoul 120-752, south Korea. Tel: +82 222280879 ; Fax: +82 2 3131894; E-mail: hkim1@yuhs.ac

or S Ramakrishna, Graduate School of Biomedical Science and Engineering, Hanyang University, Seoul 133-791, South Korea. Tel: +82 222202424 ; Fax: +82 222202422 ; E-mail: suresh.ramakris@gmail.com or suri28@ hanyang.ac.kr

${ }^{5}$ These authors contributed equally to this work.

Abbreviations: PTM, post-translational modification; DUB, deubiquitinating enzyme; ICM, inner cell mass; ESCs, embryonic stem cells; UCH, ubiquitin C-terminal hydrolase; USP, ubiquitin-specific protease; REST, repressor element 1-silencing transcription factor; Hes1, hairy and enhancer of split 1; iPSCs, induced pluripotent stem cells; EMT, epithelial - mesenchymal transition

Received 21.11.15; revised 16.4.16; accepted 18.4.16; Edited by JP Medema; published online 10.6.16
} 
A few studies have indicated that PTMs such as phosphorylation and sumoylation contribute to the spatial and temporal regulation of pluripotency-associated transcriptional networks. ${ }^{2,3}$ However, the roles of ubiquitin-proteasomal pathway-mediated protein turnover and the counterpart of this pathway, deubiquitination, on pluripotency-related transcriptional networks are poorly understood. Recent studies have provided strong evidence that deubiquitinating enzymes (DUBs) are important for the maintenance of stemness and stem cell differentiation.

\section{Ubiquitin-Proteasome Pathway}

Ubiquitination is a PTM in which ubiquitin is conjugated to a protein substrate, thereby regulating the stability and activity of the modified protein. For a ubiquitin molecule to be attached to a target protein, the sequential actions of three different classes of enzymes - E1 (ubiquitin activating enzymes), E2 (ubiquitin conjugating enzymes), and E3 (ubiquitin ligases) are required. Initially, the ubiquitin molecule is activated by an E1 enzyme through an ATP-dependent reaction. This activation is then followed by conjugation via an E2 class enzyme, after which an E3 ubiquitin ligase transfers the ubiquitin molecule specifically to its target protein ${ }^{4-6}$ (Figure 1).

Ubiquitin is a small and highly conserved 76-amino acid protein with a molecular weight of $8.5 \mathrm{kDa}$. The ubiquitin modification can be covalently attached to protein substrates as either a monomer or as a polymer. ${ }^{7,8}$ The different ubiquitin modifications depend on the type of chain formed during the process. ${ }^{9}$ Substrate proteins can be modified with monoubiquitin, multiple monoubiquitin (multi-ubiquitination), or a polyubiquitin chain (polyubiquitination) (Figure 2). During polyubiquitination, any of the seven lysine $(K)$ residues $(K 6$, $\mathrm{K} 11, \mathrm{~K} 27, \mathrm{~K} 29$, K33, K48, and $\mathrm{K} 63$ ) of ubiquitin can be utilized for the formation of ubiquitin - ubiquitin linkages, resulting in a sizeable chain increase with different configurations called polyubiquitin chains. ${ }^{10}$

Generally, protein substrates that undergo monoubiquitination are involved in DNA repair, vesicle sorting, receptor endocytosis, or signal transduction. ${ }^{11-14}$ On the other hand, $\mathrm{K} 6, \mathrm{~K} 11, \mathrm{~K} 29$, and $\mathrm{K} 48$ polyubiquitin chains regulate protein stability. Specifically, K48 polyubiquitin chains target proteins for proteolysis through the $26 \mathrm{~S}$ proteasome, ${ }^{15-17} \mathrm{~K} 11$ polyubiquitin chains regulate endoplasmic reticulum-mediated degradation and cell cycle progression, ${ }^{18-20} \mathrm{~K} 63$ polyubiquitin chains signal activation of the transcription factor nuclear factor- $\kappa B$ or regulate the DNA repair process, ${ }^{21-24}$ and K29 chains are involved in lysosomal degradation. ${ }^{25}$ However, the roles of other polyUb chains are not well understood, in spite of a few intriguing reports. K29 or K33 chains are found to bind AMPK-related kinases to regulate their enzymatic activity, ${ }^{26} \mathrm{~K} 6$ chain linkages are induced by BRCA1/BARD1 E3 and speculated to be involved in the DNA repair process, ${ }^{27,28}$ and $\mathrm{K} 27$ and K33 chains may be assembled by U-box-type E3 ligases during a stress response $e^{29,30}$ (Figure 2). However, ubiquitination is also involved in diverse cellular functions such as transcriptional regulation, the immune response, apoptosis, cell cycle control, oncogenesis, embryonic development, preimplantation, and intracellular signaling pathways. ${ }^{31}$

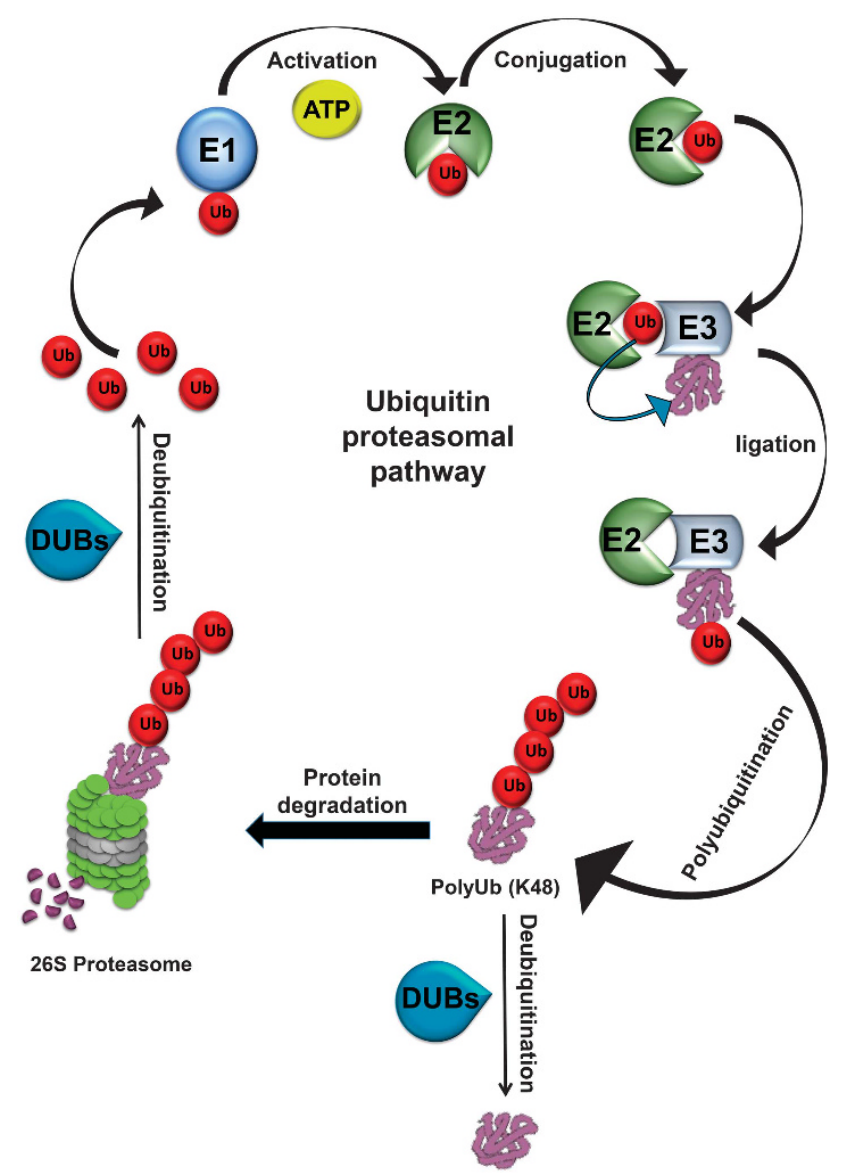

Figure 1 The ubiquitin proteasomal pathway. Ubiquitin molecule to be attached to a target protein, the sequential actions of three different classes of enzymes - E1 (ubiquitin-activating enzymes), E2 (ubiquitin-conjugating enzymes), and E3 (ubiquitin ligases) - promote the ligation of a ubiquitin molecule to the lysine $(K)$ residues in the protein substrates. K48-linked polyubiquitination chain-attached protein substrates are targeted to the $26 \mathrm{~S}$ proteasome for protein degradation. Ubiquitins are recycled by the action of DUBs through the ubiquitin-proteasome pathway

\section{Deubiquitination}

The DUBs comprise a class of proteases that cleave ubiquitin molecules from ubiquitin-conjugated protein substrates. Specifically, DUBs selectively cleave the isopeptide bond present at the ubiquitin C-terminus., ${ }^{9,32}$ DUBs prevent proteasomedependent and lysosome-dependent protein degradation because they counteract E3 ligase-mediated ubiquitination. Consequently, DUBs indirectly alter the activities and levels of their target proteins.

Deubiquitinating enzymes and their classifications. DUBs can be classified into six families: (i) ubiquitin C-terminal hydrolases (UCHs), (ii) ubiquitin-specific proteases (USPS), (iii) Jab1/Pab1/MPN domain-containing metallo-enzymes, (iv) otu-domain ubiquitin aldehyde-binding proteins, (v) Ataxin-3/Josephin proteases, and (vi) monocyte chemotactic protein-induced proteases. Of these families, the USP family is the largest. This family is comprised of more than 50 members, each of which contains conserved domains and catalytic sites. $3,4,33-37$ 


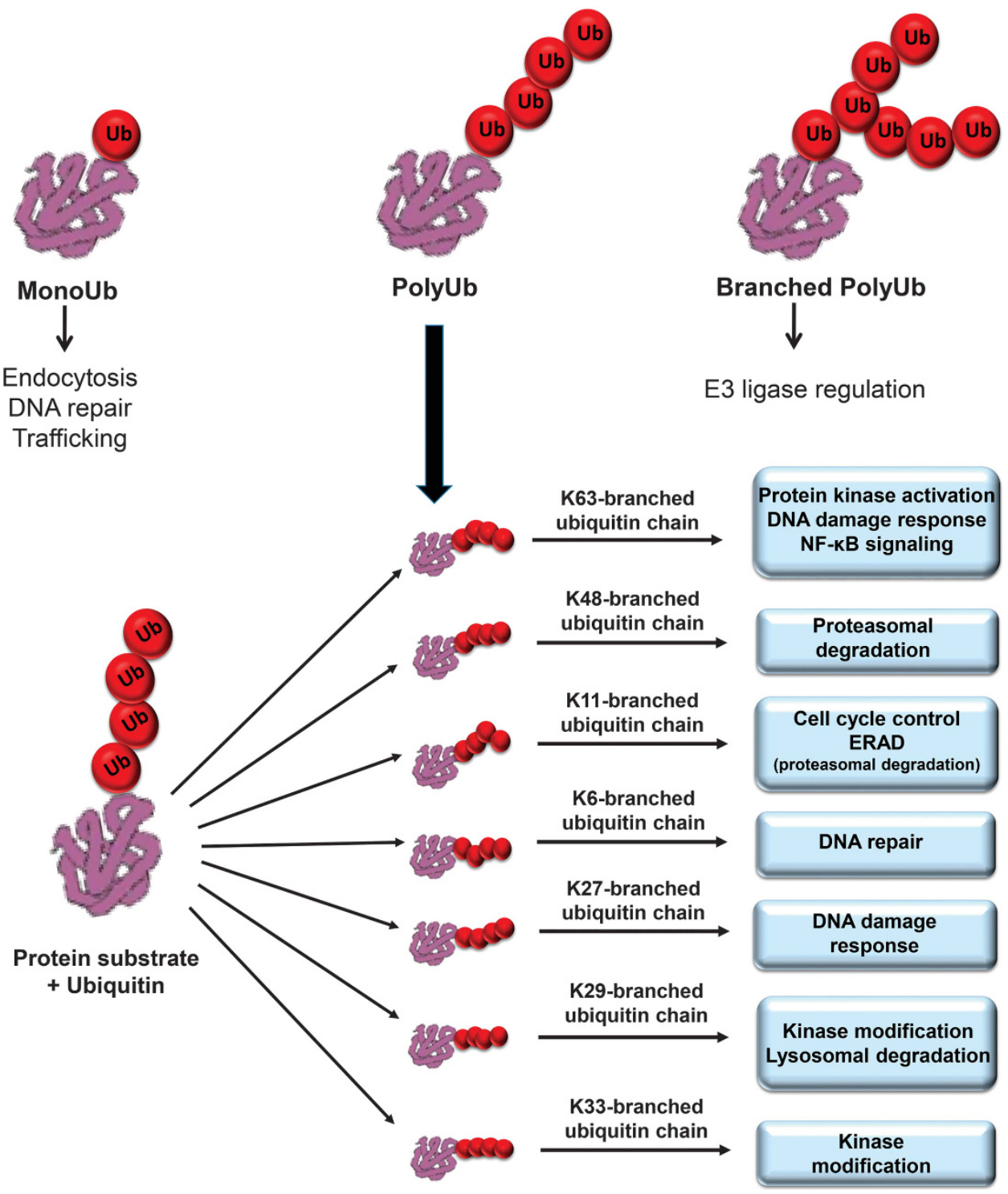

Figure 2 Different forms of polyubiquitination and their cellular functions. Protein substrates can be monoubiquitinated or polyubiquitinated. The attachment of ubiquitin molecules to one or more lysine $(K)$ residues results in polyubiquitination. Different forms of polyubiquitin chains linked via K-residues on the protein substrate are implicated in diverse cellular functions

Deubiquitinating enzymes functions. DUBs have four distinct mechanisms of action: (i) processing of ubiquitin precursors/de novo ubiquitin synthesis, (ii) recycling of ubiquitin molecules during ubiquitination, (iii) cleavage of polyubiquitin chains, and (iv) reversal of ubiquitin conjugation. ${ }^{4,38}$ Through these actions, DUBs are critical regulators of the proteasomal pathway.

DUBs regulate several cellular functions such as proteasomedependent and lysosome-dependent proteolysis, gene expression, cell cycle progression, chromosome segregation, kinase activation, apoptosis, localization, DNA repair, spermatogenesis, and degradation of signaling intermediates. ${ }^{3,4,36-39}$

\section{Deubiquitinating Enzymes in Stem Cells}

All stem cells possess two defining characteristics, the ability to self-renew and the ability to differentiate. ESCs maintain high-genomic plasticity and can therefore enter any differentiation pathway. However, ESC differentiation is mainly regulated by the turnover of transcription factors such as Oct3/4, Sox2, KIf4, c-Myc, Nanog, LIN28, and Sall4. These transcription factors are master regulators of stem cell pluripotency. ${ }^{3,40,41}$ A growing body of evidence supports the idea that UPSs are important for stem cell pluripotency and differentiation. ${ }^{2,3,40}$ Achieving the appropriate UPS expression levels and subcellular localizations is critical for maintaining stem cell pluripotency. ${ }^{40}$ Although UPSs have been reported to have a number of physiological functions related to ESC pluripotency, only limited information is available regarding DUB function in stem cell maintenance and differentiation. However, recent studies with USP7, USP9X, USP22, USP44, and Psmd14 have shown that DUBs are involved in maintaining stem cell pluripotency. We will now discuss the published 
evidence and current knowledge regarding DUB function and the contribution of DUBs to stem cell maintenance and differentiation.

Ubiquitin-specific protease 7. Herpesvirus-associated ubiquitin-specific protease, also known as ubiquitin-specific protease 7 (USP7), was initially identified via its association with the viral protein ICPO (herpes simplex virus type 1 regulatory protein) and was shown to regulate its stability. ${ }^{42}$ USP7 was also found to regulate the transcriptional activity of Epstein - Barr nuclear antigen $1 .{ }^{43}$ Although USP7 is involved in various cellular processes, ${ }^{44}$ it was recently shown to prevent the degradation of repressor element 1-silencing transcription factor (REST) through its deubiquitinating activity, thereby facilitating the maintenance of neural stem/progenitor cells. ${ }^{45}$ REST is a stem cell transcription factor whose protein level is altered during neural differentiation. REST is targeted for ubiquitindependent protein degradation via the $\mathrm{SCF}^{\beta-\operatorname{TrCP}} \mathrm{E} 3$ ubiquitin ligase complex. USP7 interacts with and stabilizes REST by preventing $\mathrm{SCF}^{\beta-\mathrm{TrCP}}$-mediated ubiquitination, thus promoting the maintenance of stemness. ${ }^{45}$

Ubiquitin-specific protease $\mathbf{9 X}$. USP9X is one of the largest members of the USP family and was originally identified in Drosophila; mutations in USP9X cause characteristic eye defects called fat facets. ${ }^{46,47}$ In mammals, USP9 is known as FAM. ${ }^{48}$ Several stemness-related genes are highly expressed in stem cells compared with their differentiated progeny. Of these genes, USP9X has been shown to be highly expressed in stem cells in vivo, including pre-implantation blastomere embryos and neural stem cells. ${ }^{49-51}$ USP9X has also been identified in mouse and human stem cells, including ESCs, neural stem cells, neuronal progenitors, hematopoietic stem cells, and adult epidermal stem cells. ${ }^{52,53}$ Although inhibition of USP9X in mouse ESCs did not affect their growth in vitro, ${ }^{54}$ USP9X has been hypothesized to regulate the early differentiation of stem cells. Interestingly, USP9X has been found to regulate the mTOR pathway, thus controlling the proliferation and differentiation of muscle stem cells. ${ }^{55}$ Although USP9X is highly expressed in neural stem cells, its expression in adult brain tissue is significantly decreased. ${ }^{50,51}$ However, USP9X expression is maintained in the neural progenitors located in the adult neurogenic niches. ${ }^{50,51}$ Thus, USP9X expression is critical for stem cell function.

Ubiquitin-specific protease 22. USP22 is a deubiquitinating subunit of the SAGA mDUB complex. ${ }^{56}$ This enzyme has been reported to affect transcription by hydrolyzing the monoubiquitin molecules that are conjugated to $\mathrm{UH} 2 \mathrm{~A}$ and uH2B. ${ }^{56-60}$ A number of studies have indicated that USP22 has an important role in tumorigenesis and tumor progression. ${ }^{61-63}$ Indeed, USP22 was initially reported as a member of an 11-gene 'death from cancer' gene expression signature characterized by high malignancy, metastatic dissemination, and resistance to therapy. ${ }^{64,65}$ In addition to its role in tumorigenesis, USP22 also has a major role in stem cell function. The USP22 locus has been shown to be actively transcribed in human ESCs and induced pluripotent stem cells (iPSCs). ${ }^{66}$ Moreover, the histone H3 lysine 4 trimethyl epigenetic mark is recruited to the USP22 promoter, which is co-occupied by the stemness factor $K L F 4$, indicating its role in stem cell pluripotency and differentiation. ${ }^{66}$

Recent evidence has indicated that USP22 regulates core pluripotency factors, including c-Myc and Sox2. ${ }^{56,67}$ USP22 was originally identified as an essential cofactor for the stem cell transcription factor Myc, thereby regulating transcription of Myc target genes. ${ }^{56}$ Moreover, Sussman et al. ${ }^{67}$ showed that USP22 is required for proper ESC differentiation into all three germ layers. During ESC differentiation, USP22 negatively regulates Sox2 transcription in ESCs. Moreover, USP22 is located directly on the Sox2 promoter, catalyzes H2B deubiquitination, and attenuates transcription of the Sox2 locus. ${ }^{67}$ Thus, USP22 has a pivotal role in differentiation by repressing Sox2 in ESCs, which allows them to transition from a selfrenewal state into lineage-specific differentiation pathways.

Usp22 is highly expressed in adult murine tissue and is also prominent at the early embryonic stages in the midbrain, forebrain, hindbrain, and dorsal root ganglia. ${ }^{68}$ Usp22 has also been reported to be essential for embryonic development in mice. Mice with genetic ablation of Usp22 exhibit early embryonic lethality at E10.5 of the postimplantation stage. ${ }^{65}$ Recently, Kosinsky et al. ${ }^{69}$ showed that Usp22-deficient mice displayed growth defects and reduced body weight. Furthermore, Usp22-deficient mice exhibited differentiation defects in the cells of the small intestine. ${ }^{69}$ Hairy and enhancer of split 1 (Hes1) expression has been found to oscillate in mouse ESCs and in neural stem cells; this oscillation contributes to the maintenance of stem cell potency and differentiation fate. ${ }^{70}$ Recently, Usp22 was found to stabilize Hes1 via deubiquitination. On the other hand, knockdown of Usp22 shortened the half-life of Hes1 and triggered its rapid degradation, resulting in delayed auto-repression and dampened oscillation. In turn, neuronal differentiation was increased. ${ }^{71}$ Thus, Usp22 has a critical role in neuronal differentiation in the developing brain.

Ubiquitin-specific protease 44. USP44 has been reported to be a critical regulator of the mitotic spindle checkpoint in differentiated cells. Specifically, USP44 deubiquitinates Cdc20 and regulates anaphase initiation during mitosis. ${ }^{72}$ USP44 also mediates chromosome instability and aneuploidy. ${ }^{73}$ Recently, several lines of evidence have indicated that USP44 is involved in stem cell differentiation. For example, genome-scale location analysis revealed that USP44 is a direct target of Oct4. ${ }^{74}$ In addition, USP44 is localized in the nucleus, where it associates with chromatin; ${ }^{72,75}$ moreover, USP44 is downregulated during ESC differentiation. ${ }^{76}$

Recently, Fuchs et al. ${ }^{76}$ showed that USP44 directly regulates stem cell differentiation. Specifically, monoubiquitination of histone H2B on lysine 120 (H2Bub1) increases during differentiation of human and mouse ESCs; similar effects were observed in embryonic carcinoma cells. RNF20, an E3 ligase, regulates $\mathrm{H} 2 \mathrm{~B}$ ubiquitination during ESC differentiation. Genetic silencing of USP44 during ESC differentiation also resulted in increased levels of H2Bub1, suggesting that USP44 is a negative regulator of $\mathrm{H} 2 \mathrm{~B}$ ubiquitination. $^{76}$ Thus, ESC differentiation requires that USP44 is expressed at an optimum level. 
Psmd14. Psmd14 is a component of the 195 proteasome lid, which also includes Psmd3, Psmd6, Psmd7, Psmd11, Psmd12, and Psmd13. ${ }^{77}$ Although Psmd14 is highly expressed in pluripotent ESCs, its expression decreases during differentiation. Buckley et al. ${ }^{40}$ performed a UPStargeted siRNA screen to identify essential genes required for stem cell maintenance. This screen identified two DUBs, Psmd14 and USP9X. Depletion of Psmd14 is accompanied by a significant decrease in the level of Oct4 and abnormal ESC morphology. Although knockdown of Psmd14 did not affect the overall stoichiometry of the $26 \mathrm{~S}$ proteasome, proteasome activity was impaired, as evidenced by the accumulation of both K48-linked and K63-linked polyubiquitinated proteins. Moreover, MEFs expressing Psmd14-targeting shRNA failed to reprogram and generate iPSCs. ${ }^{40}$ Thus, Psmd14 is essential for the maintenance of ESC pluripotency and cellular reprogramming.

Other deubiquitinating enzymes. As discussed previously, USP7 interacts with REST, a stem cell transcription factor that regulates neuronal differentiation. ${ }^{45}$ However, REST degradation is also regulated by other DUBs, including USP14 and USP15. ${ }^{78,79}$ Treatment with IU-1, a USP14 inhibitor, resulted in decreased protein levels of REST in both in vitro and in vivo studies. ${ }^{78}$ Moreover, USP15 was found to stabilize newly synthesized REST rather than pre-existing REST and a small fraction of USP15 was found associated with polysomes. Thus, USP15 has a critical role in controlling cell cycle oscillations by facilitating rapid replenishment of newly synthesized REST upon mitotic exit, which regulates the beginning of the next cell cycle. ${ }^{79}$

\section{Deubiquitinating Enzymes as Targets for Therapeutics}

Many studies have implicated DUBs in the pathogenesis of several human diseases such as neurological disorders, cancer, and infectious diseases. ${ }^{80,81}$ Thus, DUBs are a key alternative target upstream of the proteasome ubiquitin conjugation/deconjugation system that can potentially generate more reliable, specific, and less toxic anticancer agents. In addition, recent advances in small-molecule-based inhibitors specifically targeting DUBs also make DUBs attractive therapeutic targets for antiviral and anticancer agents. ${ }^{82-84}$

Several DUBs are directly or indirectly involved in downregulating or ablating oncogene products or, alternatively, upregulating or suppressing tumor suppressors (reviewed in Lim and Baek, ${ }^{85} \mathrm{Pal}$ and Donato $\left.{ }^{86}\right)$. Some specific examples of DUBs that are viable targets for anticancer therapy include USP2, USP4, USP7, USP9X, USP11, and USP15. USP2a is a isoform of USP2 that is involved in prostate cancer, and deubiquitinates and stabilizes fatty acid synthase. ${ }^{87}$ In support of this oncogenic role, ectopic expression of USP2a in nontransformed cells promotes oncogenic signaling in vitro and in vivo. ${ }^{88}$ Elevated expression of USP7 resulting in tumor aggressiveness was reported in prostate cancer, ${ }^{89}$ whereas the absence of USP7 in nude mice led to a reduction in tumor size. $^{90}$ USP7 also inactivates several tumor suppressors including p53, FOXO4, and PTEN. ${ }^{89,91-93}$ Several DUBs are involved in regulating TGF $\beta$ signaling. Inhibition of USP4, USP11, and USP15 attenuates TGF $\beta$-mediated epithelial-mesenchymal transition (EMT) and invasion in breast cancer. ${ }^{94-96}$ Similarly, USP9X alters levels of the monoubiquitinated protein Smad4 in cells through deubiquitination, thus regulating TGF $\beta$-mediated EMT and invasion. ${ }^{97}$ USP9X was also found to interact with and deubiquitinate SMURF1, a negative regulator of TGF $\beta / B M P$ signaling during tumor cell migration and invasion. ${ }^{98,99}$ Furthermore, inhibition of USP9X leads to elevated SMURF1 protein levels resulting in SMURF1-dependent breast cancer cell motility. ${ }^{99}$ Elevated expression of USP9X is reported in human follicular lymphoma and correlates with poor prognosis in multiple myeloma. ${ }^{100}$ In addition, USP9X expression is necessary for the growth of glioblastomas and medulloblastomas. ${ }^{101}$ Taken together, these findings indicate that DUBs function as cancer-associated proteases, and their unique biochemical structures allow them to be considered as potential targets for anticancer therapies.

Recently there has been extensive research on the development of small-molecule inhibitors to target DUBs. Because DUBs have critical role in regulating cellular homeostasis, proliferation, and survival, ${ }^{36}$ they have been considered as anticancer targets. ${ }^{85,86}$ Screening for compounds that modulate the UPS has been challenging research for pharmaceutical companies. Although there are strong similarities within the active-site cysteine and histidine boxes of several DUBs, the three-dimensional structure of each DUB has unique differences in accessibility to the catalytic pocket. ${ }^{83}$ Since the approval of the proteasome inhibitors bortezomib and carfilzomib for the treatment of hematological malignancies, ${ }^{102}$ there has been great interest in targeting the deubiquitination process upstream of the proteasome in cancer therapy. Auronofin (Aur) is an inhibitor of the proteasome-associated deubiquitinases UCHL5 and USP14, but not the $20 \mathrm{~S}$ proteasome, that leads to Aur-induced cytotoxicity. Thus, Aur is more effective in inhibiting tumor growth in vivo and induces cytotoxicity in cancer cells from acute myeloid leukemia patient samples. ${ }^{103}$ Along with cancer, the applicability of DUB inhibitors is being examined in other therapeutic areas including neurodegeneration and infectious disease. ${ }^{82,83,104,105}$ Several partial and specific inhibitors against USPs have been developed and utilized in research. So far, many DUB inhibitors have been found to be effective against USP family members such as USP1, USP2, USP5, USP7, USP8, USP9x, USP10, USP11, USP13, USP14, USP20, USP30, and USP37, as well as UCH family members such as UCHL1, UCHL3, and UCHL5. ${ }^{82-84}$

The tumor suppressor p53 acts as a master regulator of stem cell and iPSC differentiation in response to genomic damage. ${ }^{106-108}$ Thus, targeting DUBs specific to p53 might be an alternative method to regulate stem cell differentiation. In response to DNA damage, p53 represses several stemnessrelated and iPSCs-associated genes including Oct4, Sox2, Nanog, Sal4, Esrb, Utf1, Prdm14, n-Myc, and c-Myc which signifies that p53 mediates transcriptional repression during ES cell differentiation and somatic cell reprogramming. ${ }^{109}$ USP10 deubiquitinates and stabilizes both wild-type and mutant p53, and reverses MDM2-mediated nuclear transport and degradation of p53. ${ }^{110}$ In addition, USP10 was found to antagonize $c-M y c$ transcription by deubiquitinating SIRT6 and NEMO.$^{111}$ Considering the importance of p53 and c-Myc in the process of reprogramming somatic cells to iPSCs, USP10 


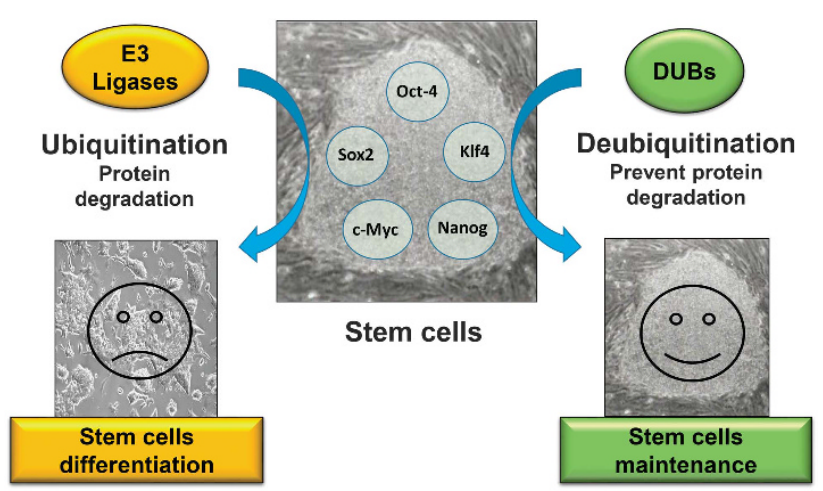

Figure 3 Roles of ubiquitination and deubiquitination in regulating stem cell differentiation and pluripotency. Ubiquitination of core stem cell transcription factors by E3 ligases drives stem cell differentiation. Deubiquitination of the core stem cell transcription factors by DUBs mediates stem pluripotency

might be a prime target during iPSCs generation. USP15 was shown to deubiquitinate and stabilize MDM2, which impacts both p53 and the T-cell transcription factor NFATc. ${ }^{112}$ Thus, USP15 is a potential candidate for targeting and analyzing the p53-mediated regulation of genome stability in stem cells. The biological functions associated with USP7 silencing and the links between USP7 and p53/Mdm2 $2^{91,92}$ strongly suggest that small-molecule inhibitor-mediated targeting of USP7 could be a highly useful therapy for the regulation of stem cell pluripotency and differentiation.

\section{Conclusions}

The determination of cell fate is exquisitely controlled by transcription factors that regulate stem cell maintenance and differentiation. Perturbation in activation of core stem cell pathways leads to transformation, whereas deficiencies in these cellular mechanisms drives to degenerative conditions. Stem cell fate results from a delicate balance between ubiquitination and deubiquitination. For instance, E3 ligases catalyze the ubiquitination of stemness-related proteins, thereby driving stem cell differentiation, whereas DUBs stabilize stemness-related proteins, thus preventing stem cell differentiation (Figure 3).

A growing body of evidence indicates that stemness-related proteins, which regulate self-renewal and stem cell maintenance, are ubiquitinated by E3 ligases. ${ }^{3,40}$ Although much attention has been given to the function of the ubiquitination system in the maintenance of pluripotency, DUB-mediated reversal of ubiquitination also has an equally critical role. DUBs are usually present in large multi-protein complexes that function directly or indirectly in stem cell pluripotency, differentiation, and reprogramming (Figure 4). For instance, Boyer et al. ${ }^{74}$ performed a genome-scale location analysis that implicated several DUBs in the transcriptional regulation of human ESCs via their binding to the promoter regions of Yamanaka factors such as Oct4, Sox2, and Nanog. Speci®cally, USP7 and USP44 bind to the Oct4 promoter; USP7, USP25, USP44, and USP49 bind to the Sox2 promoter; and USP3, USP7, USP10, USP16, USP37, and USP44 bind to the Nanog promoter (Table 1). However, the mechanism(s) by which DUBs regulate Oct4, Sox2, and Nanog, and the role of

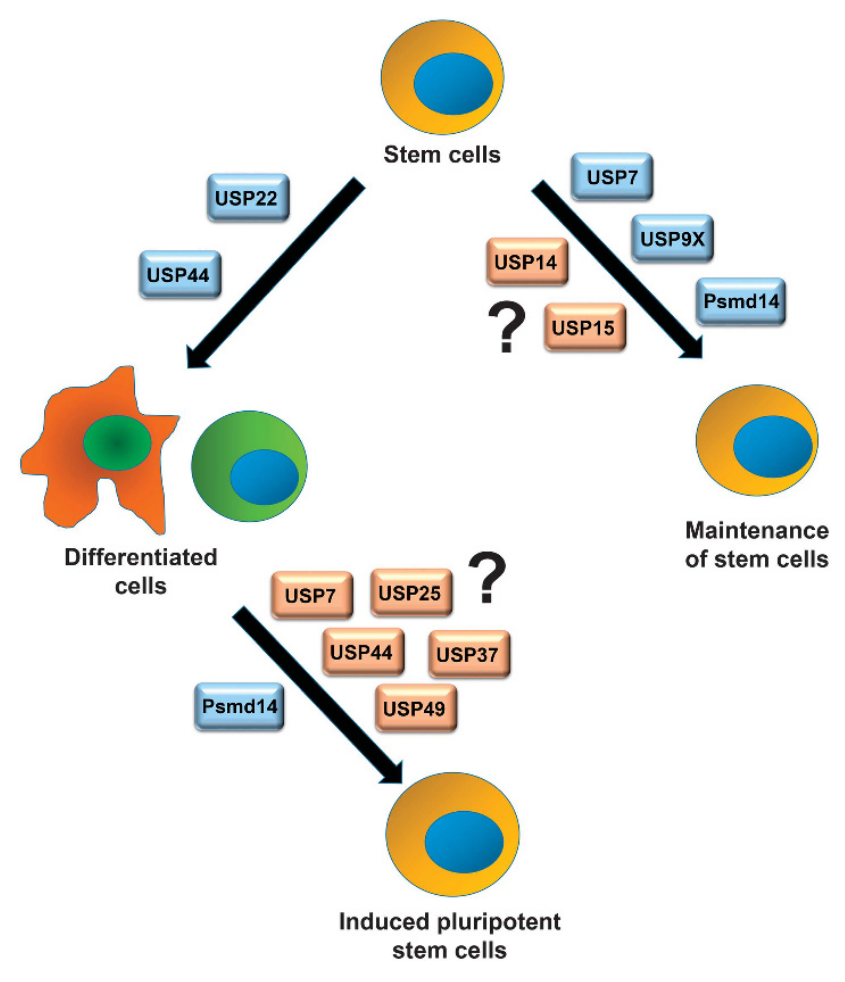

Figure 4 Roles of DUBs in regulating stem cell differentiation, pluripotency, and cellular reprogramming. Reported DUBs involved in fate determination of stem cells are colored in blue and predicted DUBs are colored in orange

Table 1 Deubiquitinating enzymes binding to Oct4, Sox2, and Nanog promoter region

\begin{tabular}{lll}
\hline Oct4 & Sox2 & Nanog \\
\hline USP7 & USP7 & USP3 \\
USP44 & USP25 & USP7 \\
& USP37 & USP10 \\
& USP44 & USP16 \\
& USP49 & USP37 \\
& & USP44 \\
\hline
\end{tabular}

USP, ubiquitin-specific protease

DUBs in stem cell differentiation and cellular reprogramming have not yet been elucidated.

All work to this point indicates that Yamanaka factors are specifically deubiquitinated by multiple DUBs. Thus, reprogramming efficiency could be improved by screening for specific DUBs that regulate the levels of stem cell transcription factors. Moreover, reprogramming could be optimized by overexpressing a combination of DUBs that stabilize stemness-related proteins and certain Yamanaka factors, which could theoretically improve the efficiency of generating iPSCs. Therefore, screens for specific DUBs that catalyze the cleavage of ubiquitin moieties from Yamanaka factors promise to shed light on the molecular mechanisms that determine the cell fate of ESCs. In addition, screening specific DUBs for defined factors involved in the direct conversion of fibroblasts to functional neurons, ${ }^{113}$ melanocytes, ${ }^{114}$ endothelial cells, ${ }^{115}$ astrocytes, ${ }^{116}$ osteoblasts, ${ }^{117}$ hepatocytes, ${ }^{118}$ and so 
on, might have significant implications on research studies and improve the efficiency of cell fate conversion. Further detailed mapping of the cross talk between ubiquitination and deubiquitination in the context of the regulation of ESC function, pluripotency, and differentiation will also be important. We anticipate that these studies will open up exciting new avenues for future research and could also initiate the development of DUB-targeted treatment approaches for various human disorders.

\section{Conflict of Interest}

The authors declare no conflict of interest.

Acknowledgements. We would like to thank all of Suri's laboratory members for their helpful discussions. This study was supported by a grant of the National Research Foundation of Korea (201500000002885, 2015R1D1A1A01060907, and 2015H1D3A1036065 for HK).

1. Thomson JA, Itskovitz-Eldor J, Shapiro SS, Waknitz MA, Swiergiel JJ, Marshall VS et al. Embryonic stem cell lines derived from human blastocysts. Science 1998; 282: 1145-1147.

2. Cai N, Li M, Qu J, Liu GH, Izpisua Belmonte JC. Post-translational modulation of pluripotency. J Mol Cell Biol 2012; 4: 262-265.

3. Ramakrishna S, Kim KS, Baek KH. Posttranslational modifications of defined embryonic reprogramming transcription factors. Cell Reprogram 2014; 16: 108-120.

4. Amerik AY, Hochstrasser M. Mechanism and function of deubiquitinating enzymes. Biochim Biophys Acta 2004; 1695: 189-207.

5. Ciechanover A, Shkedy D, Oren M, Bercovich B. Degradation of the tumor suppressor protein p53 by the ubiquitin-mediated proteolytic system requires a novel species of ubiquitin-carrier protein, E2. J Biol Chem 1994; 269: 9582-9589.

6. Ciechanover A, Heller H, Elias S, Haas AL, Hershko A. ATP-dependent conjugation of reticulocyte proteins with the polypeptide required for protein degradation. Proc Natl Acad Sci USA 1980; 77: 1365-1368.

7. Hershko A, Ciechanover A. The ubiquitin system. Annu Rev Biochem 1998; 67: 425-479.

8. Fang S, Weissman AM. A field guide to ubiquitylation. Cell Mol Life Sci 2004; 61: 1546-1561.

9. Komander D, Rape M. The ubiquitin code. Annu Rev Biochem 2012; 81: 203-229.

10. Komander D. The emerging complexity of protein ubiquitination. Biochem Soc Trans 2009; 37(Pt 5): 937-953.

11. Sigismund S, Polo S, Di Fiore PP. Signaling through monoubiquitination. Curr Top Microbiol Immunol 2004; 286: 149-185.

12. Sun L, Chen ZJ. The novel functions of ubiquitination in signaling. Curr Opin Cell Biol 2004; 16: 119-126.

13. Sadowski M, Suryadinata R, Tan AR, Roesley SN, Sarcevic B. Protein monoubiquitination and polyubiquitination generate structural diversity to control distinct biological processes. IUBMB Life 2012; 64: 136-142.

14. Ramanathan $\mathrm{HN}, \mathrm{Ye}$ Y. Cellular strategies for making monoubiquitin signals. Crit Rev Biochem Mol Biol 2012; 47: 17-28.

15. Kim I, Mi K, Rao H. Multiple interactions of rad23 suggest a mechanism for ubiquitylated substrate delivery important in proteolysis. Mol Biol Cell 2004; 15: 3357-3365.

16. Kravtsova-Ivantsiv $Y$, Sommer $T$, Ciechanover A. The lysine48-based polyubiquitin chain proteasomal signal: not a single child anymore. Angew Chem Int Ed Engl 2013; 52: 192-198.

17. Verma R, Oania R, Graumann J, Deshaies RJ. Multiubiquitin chain receptors define a layer of substrate selectivity in the ubiquitin-proteasome system. Cell 2004; 118: 99-110.

18. Xu P, Duong DM, Seyfried NT, Cheng D, Xie Y, Robert J et al. Quantitative proteomics reveals the function of unconventional ubiquitin chains in proteasomal degradation. Cell 2009; 137: 133-145.

19. Locke M, Toth JI, Petroski MD. Lys11- and Lys48-linked ubiquitin chains interact with p97 during endoplasmic-reticulum-associated degradation. Biochem J 2014; 459: 205-216.

20. Matsumoto ML, Wickliffe KE, Dong KC, Yu C, Bosanac I, Bustos D et al. K11-linked polyubiquitination in cell cycle control revealed by a K11 linkage-specific antibody. Mol Cell 2010; 39: 477-484.

21. Chen ZJ. Ubiquitin signalling in the NF-kappaB pathway. Nat Cell Biol 2005; 7: 758-765.

22. Hoege C, Pfander B, Moldovan GL, Pyrowolakis G, Jentsch S. RAD6-dependent DNA repair is linked to modification of PCNA by ubiquitin and SUMO. Nature 2002; 419: 135-141.

23. Sims JJ, Cohen RE. Linkage-specific avidity defines the lysine 63-linked polyubiquitinbinding preference of rap80. Mol Cell 2009; 33: 775-783.

24. Tokunaga F, Sakata S, Saeki Y, Satomi Y, Kirisako T, Kamei K et al. Involvement of linear polyubiquitylation of NEMO in NF-kappaB activation. Nat Cell Biol 2009; 11: 123-132.

25. Chastagner P, Israel A, Brou C. Itch/AIP4 mediates Deltex degradation through the formation of K29-linked polyubiquitin chains. EMBO Reports 2006; 7: 1147-1153.
26. Al-Hakim AK, Zagorska A, Chapman L, Deak M, Peggie M, Alessi DR. Control of AMPKrelated kinases by USP9X and atypical Lys(29)/Lys(33)-linked polyubiquitin chains. Biochem J 2008; 411: 249-260.

27. Morris JR, Solomon E. BRCA1: BARD1 induces the formation of conjugated ubiquitin structures, dependent on $\mathrm{K} 6$ of ubiquitin, in cells during DNA replication and repair. Hum Mol Genet 2004; 13: 807-817.

28. Nishikawa H, Ooka S, Sato K, Arima K, Okamoto J, Klevit RE et al. Mass spectrometric and mutational analyses reveal Lys-6-linked polyubiquitin chains catalyzed by BRCA1-BARD1 ubiquitin ligase. J Biol Chem 2004; 279: 3916-3924.

29. Hatakeyama S, Yada M, Matsumoto M, Ishida N, Nakayama KI. U box proteins as a new family of ubiquitin-protein ligases. J Biol Chem 2001; 276: 33111-33120.

30. Gatti M, Pinato S, Maiolica A, Rocchio F, Prato MG, Aebersold R et al. RNF168 promotes noncanonical K27 ubiquitination to signal DNA damage. Cell Rep 2015; 10: 226-238.

31. Akutsu M, Dikic I, Bremm A. Ubiquitin chain diversity at a glance. J Cell Sci2016; 129 : 875-880.

32. Komander D, Clague MJ, Urbe S. Breaking the chains: structure and function of the deubiquitinases. Nat Rev Mol Cell Biol 2009; 10: 550-563.

33. Baek KH. Cytokine-regulated protein degradation by the ubiquitination system. Curr Protein Pept Sci 2006; 7: 171-177.

34. Baek KH, Kim MS, Kim YS, Shin JM, Choi KH. DUB-1A, a novel subfamily member of deubiquitinating enzyme, is polyubiquitinated and cytokine inducible in B-lymphocytes. J Biol Chem 2004; 279: 2368-2376.

35. Lim KH, Ramakrishna S, Baek KH. Molecular mechanisms and functions of cytokineinducible deubiquitinating enzymes. Cytokine Growth Factor Rev 2013; 24: 427-431.

36. Ramakrishna S, Suresh B, Baek KH. The role of deubiquitinating enzymes in apoptosis Cell Mol Life Sci 2011; 68: 15-26.

37. Ramakrishna S, Suresh B, Baek KH. Biological functions of hyaluronan and cytokineinducible deubiquitinating enzymes. Biochim Biophys Acta 2015; 1855: 83-91.

38. Reyes-Turcu FE, Ventii KH, Wilkinson KD. Regulation and cellular roles of ubiquitin-specific deubiquitinating enzymes. Annu Rev Biochem 2009; 78: 363-397.

39. Suresh B, Lee J, Hong SH, Kim KS, Ramakrishna S. The role of deubiquitinating enzymes in spermatogenesis. Cell Mol Life Sci 2015; 72: 4711-4720.

40. Buckley SM, Aranda-Orgilles B, Strikoudis A, Apostolou E, Loizou E, Moran-Crusio K et al. Regulation of pluripotency and cellular reprogramming by the ubiquitin-proteasome system. Cell Stem Cell 2012; 11: 783-798.

41. Okita Y, Nakayama KI. UPS delivers pluripotency. Cell Stem Cell 2012; 11: 728-730.

42. Boutell C, Canning M, Orr A, Everett RD. Reciprocal activities between herpes simplex virus type 1 regulatory protein ICPO, a ubiquitin E3 ligase, and ubiquitin-specific protease USP7. J Virol 2005; 79: 12342-12354.

43. Holowaty MN, Frappier L. HAUSP/USP7 as an Epstein-Barr virus target. Biochem Soc Trans 2004; 32(Pt 5): 731-732.

44. Nicholson B, Suresh Kumar KG. The multifaceted roles of USP7: new therapeutic opportunities. Cell Biochem Biophys 2011; 60: 61-68.

45. Huang Z, Wu Q, Guryanova OA, Cheng L, Shou W, Rich JN et al. Deubiquitylase HAUSP stabilizes REST and promotes maintenance of neural progenitor cells. Nat Cell Biol 2011; 13: $142-152$.

46. Taya S, Yamamoto T, Kano K, Kawano Y, Iwamatsu A, Tsuchiya T et al. The Ras target AF-6 is a substrate of the fam deubiquitinating enzyme. J Cell Biol 1998; 142: 1053-1062.

47. Chen X, Overstreet $E$, Wood SA, Fischer JA. On the conservation of function of the Drosophila fat facets deubiquitinating enzyme and Fam, its mouse homolog. Dev Genes Evol 2000; 210: 603-610.

48. Huang Y, Baker RT, Fischer-Vize JA. Control of cell fate by a deubiquitinating enzyme encoded by the fat facets gene. Science 1995; 270: 1828-1831.

49. Pantaleon M, Kanai-Azuma M, Mattick JS, Kaibuchi K, Kaye PL, Wood SA. FAM deubiquitylating enzyme is essential for preimplantation mouse embryo development. Mech Dev 2001; 109: 151-160.

50. Jolly LA, Taylor V, Wood SA. USP9X enhances the polarity and self-renewal of embryonic stem cell-derived neural progenitors. Mol Biol Cell 2009; 20: 2015-2029.

51. Stegeman S, Jolly LA, Premarathne S, Gecz J, Richards LJ, Mackay-Sim A et al. Loss of Usp9x disrupts cortical architecture, hippocampal development and TGFbeta-mediated axonogenesis. PLOS One 2013; 8: e68287.

52. Ramalho-Santos M, Yoon S, Matsuzaki Y, Mulligan RC, Melton DA. 'Stemness' transcriptional profiling of embryonic and adult stem cells. Science 2002; 298: 597-600.

53. Blanpain C, Lowry WE, Geoghegan A, Polak L, Fuchs E. Self-renewal, multipotency, and the existence of two cell populations within an epithelial stem cell niche. Cell 2004; 118: 635-648.

54. Nagai H, Noguchi T, Homma K, Katagiri K, Takeda K, Matsuzawa A et al. Ubiquitin-like sequence in ASK1 plays critical roles in the recognition and stabilization by USP9X and oxidative stress-induced cell death. Mol Cell 2009; 36: 805-818.

55. Agrawal P, Chen YT, Schilling B, Gibson BW, Hughes RE. Ubiquitin-specific peptidase 9 , X-linked (USP9X) modulates activity of mammalian target of rapamycin (mTOR). J Bio Chem 2012; 287: 21164-21175.

56. Zhang XY, Varthi M, Sykes SM, Phillips C, Warzecha C, Zhu W et al. The putative cance stem cell marker USP22 is a subunit of the human SAGA complex required for activated transcription and cell-cycle progression. Mol Cell 2008; 29: 102-111.

57. Henry KW, Wyce A, Lo WS, Duggan LJ, Emre NC, Kao CF et al. Transcriptional activation via sequential histone H2B ubiquitylation and deubiquitylation, mediated by SAGAassociated Ubp8. Genes Dev 2003; 17: 2648-2663. 
58. Kohler A, Zimmerman E, Schneider M, Hurt E, Zheng N. Structural basis for assembly and activation of the heterotetrameric SAGA histone H2B deubiquitinase module. Cell 2010; 141: 606-617.

59. Zhang XY, Pfeiffer HK, Thorne AW, McMahon SB. USP22, an hSAGA subunit and potential cancer stem cell marker, reverses the polycomb-catalyzed ubiquitylation of histone $\mathrm{H} 2 \mathrm{~A}$. Cell Cycle 2008; 7: 1522-1524.

60. Zhao Y, Lang G, Ito S, Bonnet J, Metzger E, Sawatsubashi S et al. ATFTC/STAGA module mediates histone $\mathrm{H} 2 \mathrm{~A}$ and $\mathrm{H} 2 \mathrm{~B}$ deubiquitination, coactivates nuclear receptors, and counteracts heterochromatin silencing. Mol Cell 2008; 29: 92-101.

61. Atanassov BS, Dent SY. USP22 regulates cell proliferation by deubiquitinating the transcriptional regulator FBP1. EMBO Reports 2011; 12: 924-930.

62. Liu YL, Jiang SX, Yang YM, Xu H, Liu JL, Wang XS. USP22 acts as an oncogene by the activation of BMI-1-mediated INK4a/ARF pathway and Akt pathway. Cell Biochem Biophys 2012; 62: 229-235.

63. Piao S, Liu Y, Hu J, Guo F, Ma J, Sun Y et al. USP22 is useful as a novel molecular marker for predicting disease progression and patient prognosis of oral squamous cell carcinoma. PLoS One 2012; 7: e42540.

64. Glinsky GV. Genomic models of metastatic cancer: functional analysis of death-fromcancer signature genes reveals aneuploid, anoikis-resistant, metastasis-enabling phenotype with altered cell cycle control and activated Polycomb Group (PcG) protein chromatin silencing pathway. Cell Cycle 2006; 5: 1208-1216.

65. Lin Z, Yang H, Kong Q, Li J, Lee SM, Gao B et al. USP22 antagonizes p53 transcriptional activation by deubiquitinating Sirt1 to suppress cell apoptosis and is required for mouse embryonic development. Mol Cell 2012; 46: 484-494.

66. Sridharan R, Tchieu J, Mason MJ, Yachechko R, Kuoy E, Horvath $S$ et al. Role of the murine reprogramming factors in the induction of pluripotency. Cell 2009; 136: 364-377.

67. Sussman RT, Stanek TJ, Esteso P, Gearhart JD, Knudsen KE, McMahon SB. The epigenetic modifier ubiquitin-specific protease 22 (USP22) regulates embryonic stem cell differentiation via transcriptional repression of sex-determining region Y-box 2 (SOX2). J Biol Chem 2013; 288: 24234-24246.

68. Lee HJ, Kim MS, Shin JM, Park TJ, Chung HM, Baek KH. The expression patterns of deubiquitinating enzymes, USP22 and Usp22. Gene Expr Patterns 2006; 6: 277-284.

69. Kosinsky RL, Wegwitz F, Hellbach N, Dobbelstein M, Mansouri A, Vogel T et al. Usp22 deficiency impairs intestinal epithelial lineage specification in vivo. Oncotarget 2015; 6 . 37906-37918.

70. Imayoshi I, Isomura A, Harima Y, Kawaguchi K, Kori H, Miyachi H et al. Oscillatory control of factors determining multipotency and fate in mouse neural progenitors. Science 2013; 342: $1203-1208$.

71. Kobayashi T, Iwamoto $\mathrm{Y}$, Takashima K, Isomura A, Kosodo $\mathrm{Y}$, Kawakami K et al. Deubiquitinating enzymes regulate Hes1 stability and neuronal differentiation. FEBS J 2015; 282: 2411-2423

72. Stegmeier F, Rape M, Draviam VM, Nalepa G, Sowa ME, Ang XL et al. Anaphase initiation is regulated by antagonistic ubiquitination and deubiquitination activities. Nature 2007; 446 : 876-881.

73. Zhang Y, van Deursen J, Galardy PJ. Overexpression of ubiquitin specific protease 44 (USP44) induces chromosomal instability and is frequently observed in human T-cell leukemia. PLoS One 2011; 6: e23389.

74. Boyer LA, Lee TI, Cole MF, Johnstone SE, Levine SS, Zucker JP et al. Core transcriptional regulatory circuitry in human embryonic stem cells. Cell 2005; 122: 947-956.

75. Suresh B, Ramakrishna S, Lee HJ, Choi JH, Kim JY, Ahn WS et al. K48- and K63-linked polyubiquitination of deubiquitinating enzyme USP44. Cell Biol Int 2010; 34: 799-808.

76. Fuchs G, Shema E, Vesterman R, Kotler E, Wolchinsky Z, Wilder S et al. RNF20 and USP44 regulate stem cell differentiation by modulating H2B monoubiquitylation. Mol Cell 2012; 46: 662-673.

77. Lander GC, Estrin E, Matyskiela ME, Bashore C, Nogales E, Martin A. Complete subunit architecture of the proteasome regulatory particle. Nature 2012; 482: 186-191.

78. Doeppner TR, Doehring M, Bretschneider E, Zechariah A, Kaltwasser B, Muller B et al. MicroRNA-124 protects against focal cerebral ischemia via mechanisms involving Usp14dependent REST degradation. Acta Neuropathol 2013; 126: 251-265.

79. Faronato M, Patel V, Darling S, Dearden L, Clague MJ, Urbe S et al. The deubiquitylase USP15 stabilizes newly synthesized REST and rescues its expression at mitotic exit. Cell Cycle 2013; 12: 1964-1977.

80. Colland F. The therapeutic potential of deubiquitinating enzyme inhibitors. Biochem Soc Trans 2010; 38(Pt 1): 137-143.

81. Daviet L, Colland F. Targeting ubiquitin specific proteases for drug discovery. Biochimie 2008; 90: 270-283.

82. Farshi P, Deshmukh RR, Nwankwo JO, Arkwright RT, Cvek B, Liu J et al. Deubiquitinases (DUBs) and DUB inhibitors: a patent review. Expert Opin Ther Pat 2015; 25: 1191-1208.

83. Kemp M. Recent advances in the discovery of deubiquitinating enzyme inhibitors. Prog Med Chem 2016; 55: 149-192.

84. Wei R, Liu X, Yu W, Yang T, Cai W, Liu J et al. Deubiquitinases in cancer. Oncotarget 2015; 6: $12872-12889$.

85. Lim KH, Baek KH. Deubiquitinating enzymes as therapeutic targets in cancer. Curr Pharm Des 2013; 19: 4039-4052.

86. Pal A, Donato NJ. Ubiquitin-specific proteases as therapeutic targets for the treatment of breast cancer. Breast Cancer Res 2014; 16: 461.
87. Graner E, Tang D, Rossi S, Baron A, Migita T, Weinstein LJ et al. The isopeptidase USP2a regulates the stability of fatty acid synthase in prostate cancer. Cancer Cell 2004; 5 : 253-261.

88. Priolo C, Tang D, Brahamandan M, Benassi B, Sicinska E, Ogino S et al. The isopeptidase USP2a protects human prostate cancer from apoptosis. Cancer Res 2006; 66: 8625-8632.

89. Song MS, Salmena L, Carracedo A, Egia A, Lo-Coco F, Teruya-Feldstein J et al. The deubiquitinylation and localization of PTEN are regulated by a HAUSP-PML network. Nature 2008; 455: 813-817.

90. Becker K, Marchenko ND, Palacios G, Moll UM. A role of HAUSP in tumor suppression in a human colon carcinoma xenograft model. Cell Cycle 2008; 7: 1205-1213.

91. Li M, Brooks CL, Kon N, Gu W. A dynamic role of HAUSP in the p53-Mdm2 pathway. Mol Cell 2004; 13: 879-886.

92. Li M, Chen D, Shiloh A, Luo J, Nikolaev AY, Qin J et al. Deubiquitination of $p 53$ by HAUSP is an important pathway for p53 stabilization. Nature 2002; 416: 648-653.

93. van der Horst $A$, de Vries-Smits $A M$, Brenkman $A B$, van Triest $M H$, van den Broek N, Colland $\mathrm{F}$ et al. FOXO4 transcriptional activity is regulated by monoubiquitination and USP7/HAUSP. Nat Cell Biol 2006; 8: 1064-1073.

94. Al-Salihi MA, Herhaus L, Macartney T, Sapkota GP. USP11 augments TGFbeta signalling by deubiquitylating ALK5. Open Biol 2012; 2: 120063.

95. Eichhorn PJ, Rodon L, Gonzalez-Junca A, Dirac A, Gili M, Martinez-Saez E et al. USP15 stabilizes TGF-beta receptor I and promotes oncogenesis through the activation of TGFbeta signaling in glioblastoma. Nat Med 2012; 18: 429-435.

96. Zhang L, Zhou F, Drabsch Y, Gao R, Snaar-Jagalska BE, Mickanin C et al. USP4 is regulated by AKT phosphorylation and directly deubiquitylates TGF-beta type I receptor. Nat Cell Biol 2012; 14: 717-726.

97. Dupont S, Mamidi A, Cordenonsi M, Montagner M, Zacchigna L, Adorno M et al. FAM/ USP9x, a deubiquitinating enzyme essential for TGFbeta signaling, controls Smad4 monoubiquitination. Cell 2009; 136: 123-135.

98. Ebisawa T, Fukuchi M, Murakami G, Chiba T, Tanaka K, Imamura T et al. Smurf1 interacts with transforming growth factor-beta type I receptor through $\mathrm{Smad7}$ and induces receptor degradation. J Biol Chem 2001; 276: 12477-12480.

99. Xie Y, Avello M, Schirle M, McWhinnie E, Feng Y, Bric-Furlong E et al. Deubiquitinase FAM/ USP9X interacts with the E3 ubiquitin ligase SMURF1 protein and protects it from ligase activity-dependent self-degradation. J Biol Chem 2013; 288: 2976-2985.

100. Schwickart M, Huang X, Lill JR, Liu J, Ferrando R, French DM et al. Deubiquitinase USP9X stabilizes MCL1 and promotes tumour cell survival. Nature 2010; 463: 103-107.

101. Cox JL, Wilder PJ, Gilmore JM, Wuebben EL, Washburn MP, Rizzino A. The SOX2interactome in brain cancer cells identifies the requirement of MSI2 and USP9X for the growth of brain tumor cells. PLoS One 2013; 8: e62857.

102. Kisselev AF, van der Linden WA, Overkleeft HS. Proteasome inhibitors: an expanding army attacking a unique target. Chem Biol 2012; 19: 99-115.

103. Liu N, Li X, Huang H, Zhao C, Liao S, Yang $\mathrm{C}$ et al. Clinically used antirheumatic agent auranofin is a proteasomal deubiquitinase inhibitor and inhibits tumor growth. Oncotarget 2014; 5: 5453-5471.

104. Clague MJ, Barsukov I, Coulson JM, Liu H, Rigden DJ, Urbe S. Deubiquitylases from genes to organism. Physiol Rev 2013; 93: 1289-1315.

105. Edelmann MJ, Nicholson B, Kessler BM. Pharmacological targets in the ubiquitin system offer new ways of treating cancer, neurodegenerative disorders and infectious diseases. Expert Rev Mol Med 2011; 13: e35.

106. Solozobova V, Blattner C. p53 in stem cells. World J Biol Chem 2011; 2: 202-214

107. Spike BT, Wahl GM. p53, stem cells, and reprogramming: tumor suppression beyond guarding the genome. Genes Cancer 2011; 2: 404-419.

108. Kawamura T, Suzuki J, Wang YV, Menendez S, Morera LB, Raya A et al. Linking the p53 tumour suppressor pathway to somatic cell reprogramming. Nature 2009; 460: 1140-1144.

109. Li M, He Y, Dubois W, Wu X, Shi J, Huang J. Distinct regulatory mechanisms and functions for p53-activated and p53-repressed DNA damage response genes in embryonic stem cells. Mol Cell 2012; 46: 30-42.

110. Yuan J, Luo K, Zhang L, Cheville JC, Lou Z. USP10 regulates p53 localization and stability by deubiquitinating p53. Cell 2010; 140: 384-396.

111. Niu J, Shi Y, Xue J, Miao R, Huang S, Wang T et al. USP10 inhibits genotoxic NF-kappaB activation by MCPIP1-facilitated deubiquitination of NEMO. EMBO J 2013; 32: 3206-3219.

112. Zou Q, Jin J, Hu H, Li HS, Romano S, Xiao Y et al. USP15 stabilizes MDM2 to mediate cancer-cell survival and inhibit antitumor T cell responses. Nat Immunol 2014; 15: 562-570.

113. Vierbuchen T, Ostermeier A, Pang ZP, Kokubu Y, Sudhof TC, Wernig M. Direct conversion of fibroblasts to functional neurons by defined factors. Nature 2010; 463: 1035-1041.

114. Yang R, Zheng Y, Li L, Liu S, Burrows M, Wei Z et al. Direct conversion of mouse and human fibroblasts to functional melanocytes by defined factors. Nat Commun 2014; 5: 5807

115. Han JK, Chang SH, Cho HJ, Choi SB, Ahn HS, Lee J et al. Direct conversion of adult skin fibroblasts to endothelial cells by defined factors. Circulation 2014; 130: 1168-1178.

116. Caiazzo M, Giannelli S, Valente P, Lignani G, Carissimo A, Sessa A et al. Direct conversion of fibroblasts into functional astrocytes by defined transcription factors. Stem Cell Reports 2015; 4: 25-36.

117. Yamamoto K, Kishida T, Sato Y, Nishioka K, Ejima A, Fujiwara H et al. Direct conversion of human fibroblasts into functional osteoblasts by defined factors. Proc Natl Acad Sci USA 2015; 112: 6152-6157.

118. Sekiya S, Suzuki A. Direct conversion of mouse fibroblasts to hepatocyte-like cells by defined factors. Nature 2011; 475: 390-393. 